\title{
Validação da versão brasileira do World Health Organization Disability Assessment Schedule 2.0 em idosos institucionalizados
}

Validation of the Brazilian version of the World Health Organization Disability Assessment Schedule 2.0 for institutionalized older adults

Validación de la versión brasileña del World Health Organization Disability Assessment Schedule 2.0 en ancianos institucionalizados

Thais Cristina Grou', Shamyr Sulyvan de Castro², Camila Ferreira Leite ${ }^{3}$, Mariana Thays Carvalho ${ }^{4}$, Lislei Jorge Patrizzi ${ }^{5}$

RESUMO | Fatores associados à institucionalização de idosos podem comprometer a funcionalidade desta população. São escassos os instrumentos que avaliam esse componente conforme o modelo biopsicossocial. Com vistas a medir a funcionalidade e a incapacidade, a Organização Mundial da Saúde (OMS) desenvolveu o World Health Organization Disability Assessment Schedule (WHODAS 2.0) amparada no modelo teórico-conceitual da Classificação Internacional de Funcionalidade, Incapacidade e Saúde (CIF). O objetivo deste estudo foi verificar as propriedades de medida (confiabilidade, consistência interna e validade de critério) da versão brasileira do WHODAS 2.0 em idosos institucionalizados com diferentes condições de saúde. Cem idosos participaram do estudo. A consistência interna foi avaliada pelo alfa de Cronbach. O coeficiente de Spearman foi utilizado para analisar a confiabilidade teste-reteste, com a reaplicação do questionário WHODAS após sete dias da primeira entrevista. A validade de critério (convergente e divergente) foi analisada pelo coeficiente de Spearman através da correlação dos domínios do WHODAS com os domínios do WHOQoL-bref e WHOQoLold, que avaliam qualidade de vida. Como resultado, obtivemos consistência interna adequada para todos os domínios (alfa de Cronbach $\geq 0,75$ ) e forte confiabilidade de teste-reteste $(r>0,85)$. Na validade de critério, obtivemos apenas correlações moderadas com o WHOQoL-bref e WHOQoL-old ( $r=-0,62 ; \quad r=-0,61$ respectivamente). Esses resultados mostraram que o WHODAS 2.0 é um instrumento válido e confiável como ferramenta de avaliação da funcionalidadepara idosos institucionalizados ao seguir os mesmos conceitos e princípios propostos pela OMS.

Descritores | Estudos de Validação; Avaliação da Deficiência; Saúde do Idoso Institucionalizado; Classificação Internacional de Funcionalidade, Incapacidade e Saúde; Inquéritos e Questionários.

\begin{abstract}
Factors associated with the institutionalization of older adults may interfere with the functioning of this population. There are few instruments that evaluate this component according to the biopsychosocial model. The World Health Organization Disability Assessment Schedule (WHODAS 2.0) is an instrument developed by WHO (World Health Organization) to measure functioning and disability supported by the theoretical-conceptual model of the International Classification of Functioning, Disability and Health (ICF). This study aims to verify the reliability, internal consistency (IC) and criterion validity of the Brazilian
\end{abstract}

'Universidade Federal do Triangulo Mineiro (UFTM) - Uberaba (MG), Brasil. E-mail: thaais_pf@hotmail.com. Orcid: 0000-0003-0929-3914

2Universidade Federal do Ceará (UFC) - Fortaleza (CE), Brasil. E-mail: castross@ufc.br. Orcid: 0000-0002-2661-7899

3Universidade Federal do Ceará (UFC) - Fortaleza (CE), Brasil. E-mail: camilafl.fisio@gmail.com. Orcid: 0000-0001-6375-8845

${ }^{4}$ Universidade Federal do Triângulo Mineiro (UFTM) - Uberaba (MG), Brasil. E-mail: mary13_carvalho@hotmail.com. Orcid:

0000-0003-0469-8226

${ }^{5}$ Universidade Federal do Triângulo Mineiro (UFTM) - Uberaba (MG), Brasil. E-mail: lislei.patrizzi@uftm.edu.br. Orcid:

0000-0002-3729-7675 
version of the WHODAS 2.0 when applied to institutionalized older adults with different health conditions. In total, 100 older adults participated in the study. IC was assessed by Cronbach's alpha. Spearman's coefficient was used to analyze test-retest reliability, with the WHODAS questionnaire being reapplied seven days after the first interview. Criterion validity (convergent and divergent) was also analyzed by Spearman's coefficient by the correlation analysis with the WHOQOL-BREF and the WHOQOL-OLD. As a result, we obtained an IC that was adequate for all domains (Cronbach's alpha $\geq 0.75$ ), with strong test-retest reliability ( $r>0.85$ ). In the criterion validity, we obtained only moderate correlations of the WHODAS 2.0 domains with the WHOQOL-BREF and WHOQOL-OLD domains ( $r=-0.62$; $r=-0.61$ respectively). The psychometric properties tested indicated reliability - good internal consistency and strong test-retest reliability and qualifications correlations in the criterion value. These results demonstrated that WHODAS 2.0 is a valid and reliable instrument, thus providing a tool for assessing functioning in institutionalized older adults following the concepts and principles proposed by WHO. Keywords I Validation Study; Disability Evaluation; Health of Institutionalized Older Adults; International Classification of Functioning, Disability and Health; Surveys and Questionnaires.

RESUMEN I Los factores asociados a la institucionalización de los ancianos pueden afectar la funcionalidad de esta población. Existen pocos instrumentos que evalúan este componente según el modelo biopsicosocial. Para medir la funcionalidad y la discapacidad, la Organización Mundial de la Salud (OMS) desarrolló el World
Health Organization Disability Assessment Schedule (WHODAS 2.0) con base en el modelo teórico-conceptual de la Clasificación Internacional del Funcionamiento, de la Discapacidad y de la Salud (CIF). El objetivo de este estudio fue verificar las propiedades de medición (fiabilidad, consistencia interna y validez de criterio) de la versión brasileña de WHODAS 2.0 en ancianos institucionalizados con diferentes condiciones de salud. En el estudio participaron cien ancianos. La consistencia interna se evaluó mediante el alfa de Cronbach. Se utilizó el coeficiente de Spearman para analizar la fiabilidad test-retest, con la reaplicación del cuestionario WHODAS siete días después de la primera entrevista. Para evaluar la validez de criterio (convergente y divergente), se utilizó el coeficiente de Spearman mediante la correlación de los dominios WHODAS con los dominios de WHOQoL-bref y WHOQoL-old, que evalúan la calidad de vida. Como resultado, hubo una consistencia interna adecuada para todos los dominios (alfa de Cronbach $\geq 0,75$ ) y una gran fiabilidad test-retest $(r>0,85)$. En la validez de criterio, hubo solo correlaciones moderadas con WHOQoL-bref y WHOQoL-old $(r=-0,62 ; r=-0,61$, respectivamente). Estos resultados apuntan que WHODAS 2.0 es un instrumento válido y fiable en la evaluación de ancianos institucionalizados siguiendo los mismos conceptos y principios propuestos por la OMS.

Palabras clave| Estudio de Validación; Evaluación de la Discapacidad; Salud del Anciano Institucionalizado; Clasificación Internacional del Funcionamiento, de la Discapacidad y de la Salud; Encuestas y Cuestionarios.

\section{INTRODUÇÃO}

As mudanças no perfil etário da população, especialmente em países pobres e em desenvolvimento, não refletiram no aumento da expectativa de vida saudável ${ }^{1}$. A prevalência de agravos crônicos acrescidos a estilos de vida pouco saudáveis tem impactado na população, o que acarreta um aumento dos indicadores de incapacidade nos idosos e leva à necessidade de acompanhamento contínuo de idosos com perfil de multimorbidade e maior vulnerabilidade ou em situação de dependência ${ }^{2}$.

Diante das dificuldades para suprir as necessidades biopsicossocioculturais desses idosos, o encaminhamento para instituição de longa permanência para idosos (ILPI) emerge como a estratégia de cuidado não-familiar mais adotada atualmente ${ }^{3}$. As ILPI são instituições destinadas à moradia coletiva a longo prazo àqueles que

necessitam, que são institucionalizados em decorrência de questões relacionadas com a precariedade ou a ausência de suporte familiar e social, e/ou condições financeiras desfavoráveis ${ }^{4}$.

O modelo de funcionamento das ILPI busca valorizar a independência, preservar a autoestima e respeitar a individualidade da pessoa idosa, porém, na prática, vivencia-se a perda progressiva da autonomia e independência por parte dos idosos ${ }^{3,4}$. Esse quadro se dá devido às dificuldades em adaptar-se às novas condições, agravadas pelas comorbidades preexistentes ${ }^{5}$. Alguns fatores contribuem para o declínio da funcionalidade em idosos institucionalizados: a nutrição não satisfatória ${ }^{6}$; o alto nível de dependência e inatividade ${ }^{5}$, e o isolamento social ${ }^{1}$. É fundamental um olhar mais sensível ao observar a integridade física e psicológica do idoso, bem como seus diversos aspectos, como a autonomia e suas limitações para as atividades diárias, participação social, suporte 
familiar e/ou financeiro ${ }^{4}$. Percebe-se assim a relevância para a saúde pública da avaliação da incapacidade e funcionalidade da população idosa institucionalizada pelo modelo biopsicossocial por meio de uma linguagem unificada e padronizada ${ }^{7}$.

A Organização Mundial da Saúde (OMS) publicou a Classificação Internacional de Funcionalidade, Incapacidade e Saúde (CIF); essa classificação considera a funcionalidade como um termo que abrange todas as funções, atividades e participação, além dos fatores ambientais capazes de interagir com todos os componentes ${ }^{7}$. Amparado no modelo teóricoconceitual da CIF, a OMS também desenvolveu o World Health Organization Disability Assessment Schedule (WHODAS 2.0), ferramenta genérica adaptada transculturalmente ${ }^{8}$ que proporciona escores de incapacidade e/ou funcionalidade a partir da perspectiva do respondente, o que possibilita o direcionamento e monitoramento do impacto das intervenções em saúde ${ }^{9}$.

Segundo a literatura ${ }^{10}$, faltam instrumentos que avaliem a funcionalidade em idosos institucionalizados conforme o modelo biopsicossocial consonante com o proposto pela CIF. A disponibilização de uma ferramenta confiável, auto relatada e alinhada com a proposta da OMS para aferição da incapacidade em idosos institucionalizados seria útil ao oferecer uma abordagem da saúde e funcionalidade de maneira mais completa ${ }^{10}$. O desenho de intervenções e ações centradas no paciente também seria possível por meio dessa ferramenta, visto que o WHODAS 2.0 analisa diferentes dimensões de participação social e de atividades ${ }^{9}$, possibilitando o reconhecimento de fatores associados com a incapacidade de idosos em ILPI.

O WHODAS 2.0 já foi traduzido e validado em diferentes línguas e diferentes condições de saúde ${ }^{11,12}$. Porém se desconhece, até o momento, a publicação de estudos que validem o instrumento em sua versão brasileira para uso em idosos institucionalizados. Antes de um instrumento ser considerado apto, ele deve oferecer dados válidos e confiáveis para a avaliação de saúde da população; por unanimidade, pesquisadores defendem o uso da confiabilidade e validade como principais propriedades que validam um instrumento ${ }^{13}$. Sendo assim, o objetivo deste estudo foi verificar a confiabilidade, a consistência interna $(\mathrm{CI})$ e a validade de critério da versão brasileira do WHODAS 2.0 na aplicação em idosos institucionalizados com diferentes condições de saúde, a fim de garantir a obtenção de dados consistentes e confiáveis na investigação da funcionalidade e/ou incapacidade de idosos institucionalizados.

\section{MÉTODO}

\section{Participantes}

O estudo contou com idosos de ambos os sexos residentes de sete ILPI, da cidade de Uberaba (MG). Foi definido um número amostral de 100 participantes, visto que esse valor permite a obtenção de um intervalo de confiança de IC95\% com $\pm 0,34$ de desvio-padrão ${ }^{14}$.

Os critérios de inclusão adotados foram: idade igual ou superior a 60 anos e residirem em ILPI. Foram excluídos os idosos com comprometimento cognitivo evidenciado pelo miniexame de estado mental (MEEM), considerandose o grau de escolaridade do sujeito ${ }^{15}$; os idosos que não puderam responder às perguntas da entrevista devido à perda auditiva e/ou dificuldade de comunicação por afasia; os portadores de alguma doença ou distúrbio com algum tipo de comprometimento funcional que não seja característico do processo de envelhecimento, como comprometimento neurológico grave; e aqueles que não realizaram o reteste após sete dias. $\mathrm{O}$ termo de consentimento livre e esclarecido foi assinado pelo próprio entrevistado ou pelo representante legal.

\section{Procedimentos e coleta de dados}

Todos os entrevistadores passaram por um processo de capacitação prévia que incluiu a explicação teórica do WHODAS 2.0, a apresentação do manual e a aplicação prévia do questionário. Durante as visitas nas instalações das ILPI, a cada indivíduo foi dado privacidade e conforto em salas reservadas, a fim de proporcionar a coleta de respostas mais acuradas.

No primeiro momento, o participante recebeu orientações sobre a pesquisa. A seguir, foram coletados dados demográficos e socioeconômicos dos participantes como forma de identificação inicial. Após a aplicação do MEEM, foram utilizados três instrumentos: a versão de 36 itens do WHODAS 2.0, o World Health Organization Quality of Life versão abreviada (WHOQoL-bref) ${ }^{16}$ e o World Health Organization Quality of Life-Older Adults Module Group (WHOQoL-old) ${ }^{17}$. Para a coleta de dados, foram realizadas duas entrevistas; na segunda entrevista, apenas o WHODAS foi reaplicado, por entrevistadores diferentes e igualmente treinados ${ }^{9}$. O intervalo de sete dias 
foi respeitado entre as entrevistas, intervalo que garante boa estabilidade, tendo em vista que um período superior a este poderia impactar em qualquer mudança no quadro geral do idoso institucionalizado e um período inferior a este poderia ser impactado pelo efeito da memória do respondente 9 . O fluxograma com as etapas da coleta de dados está representado pela Figura 1. A coleta foi realizada no período de setembro de 2016 a abril de 2017 e de junho a agosto de 2019.

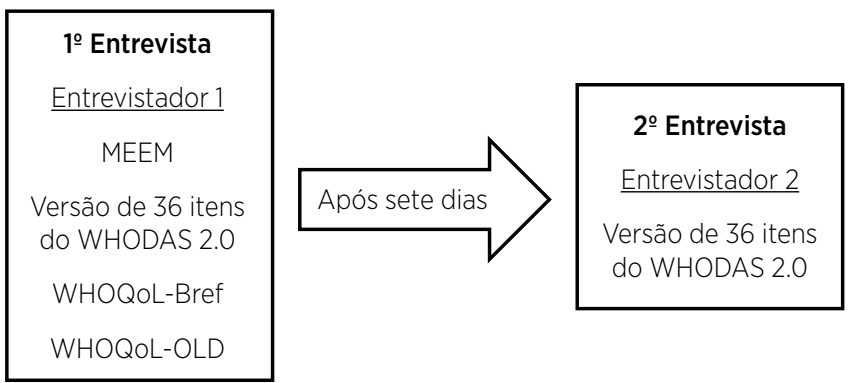

Figura 1. Fluxograma das etapas da coleta de dados

\section{Instrumentos}

Para esta pesquisa, foram utilizados os seguintes instrumentos: MEEM ${ }^{15}$; WHODAS 2.0, versão de 36 itens; WHOQoL-bref ${ }^{16}$; e WHOQoL-old ${ }^{17}$, todos aplicados por meio de entrevistas.

\section{WHODAS 2.0}

É um instrumento genérico desenvolvido pela OMS para fornecer um método padronizado de medir saúde e deficiência de forma intercultural ${ }^{8}$. Esse instrumento aborda a funcionalidade de acordo com os seguintes domínios: "cognição", com questões sobre comunicação e atividades de raciocínio; "mobilidade", que envolve atividades como ficar em pé, se movimentar dentro de casa e caminhar por longas distâncias; "autocuidado", relativo à dificuldades ao tomar banho, se vestir, comer e ficar sozinho; "relações interpessoais", que se refere às dificuldades nas interações com outras pessoas; "atividades de vida", que inclui questões sobre dificuldades nas atividades cotidianas domésticas e atividades de trabalho ou escolares; e "participação", que avalia dimensões sociais, como em atividades comunitárias, barreiras e obstáculos no ambiente à volta do respondente, além de problemas com outros assuntos como manutenção da dignidade pessoal ${ }^{9}$.

O WHODAS 2.0 possui três versões distintas, que diferem quanto ao número de itens: a versão reduzida
(12 itens); a completa (36 itens) ou a híbrida (chamada de $12+24)$. Cada pergunta tem cinco alternativas como respostas possíveis, a pontuação varia de 1 (nenhuma dificuldade) a 5 (dificuldade extrema ou não consegue fazer). O domínio e os escores gerais são computados, podendo variar de 0 a 100 (pior funcionalidade). A aplicação do WHODAS 2.0 pode ser autoadministrada, por entrevista ou administrada a um respondente substituto (proxy). No presente estudo foi utilizada versão completa, por meio de entrevista, com a utilização 32 itens do instrumento após a exclusão de quatro itens relacionados as "atividades de trabalho ou escolares”, o que é permitido quando os respondentes não desempenham estas atividades ${ }^{9}$.

\section{WHOQoL-bref}

Em sua versão em português, é um instrumento validado de rápida aplicação e útil para estudos que propõe avaliar qualidade de vida. É composto por 26 questões subdivididas em quatro domínios: "físico", "psicológico", "relações sociais" e "ambiental", com mais duas questões gerais sobre saúde e qualidade de vida. A pontuação é por meio de uma escala tipo Likert (1 a 5 pontos), na qual a maior pontuação equivale à melhor qualidade de vida ${ }^{16}$.

\section{WHOQoL-old}

Desenvolvido para a avaliação de qualidade de vida da população idosa, é capaz de ser utilizado em investigações multiculturais. Tem 24 itens em uma escala de Likert (1 a 5 pontos) e está dividido em seis domínios: "funcionamento do sensório", "autonomia", "atividades passadas, presentes e futuras", "participação social", "morte e morrer" " "intimidade". Cada domínio é composto por quatro itens, gerando um escore que pode variar de 4 a 20 pontos, convertida pela sintaxe em uma escala de 0 a 100 . Maiores escores representam melhor qualidade de vida ${ }^{17}$.

\section{MEEM}

Amplamente utilizado, permite a avaliação da função cognitiva e o rastreamento de quadros demenciais. Desde sua criação suas características psicométricas têm sido avaliadas, tanto na sua versão original quanto pelas inúmeras traduções/adaptações para várias línguas e países. Em nosso estudo, utilizamos os pontos de corte adaptados à escolaridade: 18 (analfabetos), 21 (1-3 anos de escolaridade), 24 (4-7 anos) e 26 (>7 anos) ${ }^{15}$. 


\section{Mensuração das propriedades de medida}

Para o processo de validação do WHODAS 2.0 em idosos institucionalizados, foram mensuradas três propriedades de medida: consistência interna, confiabilidade teste-reteste e validade de critério.

Consistência interna: indica se todas as subpartes de um instrumento medem a mesma característica. É um dos critérios que garantem confiabilidade, assim sendo, uma consistência interna baixa pode representar inconsistência ao medir o que foi proposto ${ }^{13}$. O coeficiente alfa de Cronbach foi utilizado para avaliar a consistência interna com agrupamento das questões em cada domínio. Considerou-se os seguintes parâmetros: 0,70-0,90: adequado; e $>0,95$ : questões redundantes ${ }^{18}$.

Confiabilidade teste-reteste: utilizada para avaliar a estabilidade do instrumento. É considerada adequada quando são obtidos resultados similares com a aplicação em dois momentos distintos após um período de tempo entre as aplicações. Como a consistência interna, faz parte dos critérios que garantem a confiabilidade ${ }^{13}$. Para esta análise, foi utilizado o coeficiente de correlação de Spearman (r). A confiabilidade teste-reteste é considerada forte quando $r$ for maior ou igual a $0,67^{19}$.

Validade de critério: é a capacidade de um instrumento em medir exatamente o que se propõe a medir ${ }^{13}$. Para este estudo, foi utilizada a validade de critério convergente e divergente. Como pressuposto, estabelecemos a hipótese relacional entre os domínios do WHODAS 2.0 com os domínios do WHOQoL-bref e do WHOQoL-old para testar a validade convergente e divergente, como representado na Tabela 1. Existe uma forte relação entre o WHOQoL e o WHODAS $2.0^{8}$. Ambos foram concebidos pela OMS e têm suas construções interligadas; no entanto, o WHODAS 2.0 avalia medidas de funcionalidade ${ }^{9}$ enquanto o WHOQL avalia medidas subjetivas de bem-estar ${ }^{16,17}$. Não é de conhecimento outro instrumento validado que trabalhe com avaliação por domínios de forma semelhante ao WHODAS 2.0. Na ausência de um instrumento "padrão-ouro", é possível testar a validade convergente por meio da correlação das pontuações do instrumento focal com os escores de outro instrumento que avalie um construto similar ${ }^{13}$. Nesse caso, os domínios de cada um dos instrumentos não são idênticos, mas se correlacionam e são similares, assim, as inferências de validade convergente e divergente são favorecidas.
Tabela 1. Hipótese correlacional entre os domínios do WHODAS e os domínios do WHOQoL-bref e WHOQoL-old

\begin{tabular}{|c|c|c|c|}
\hline WHODAS 2.0 & WHOQoL-bref & WHOQoL-old & $\begin{array}{l}\text { Relação } \\
\text { esperada }\end{array}$ \\
\hline \multirow[t]{2}{*}{ Cognição } & Psicológico & Autonomia & Convergente \\
\hline & Relações sociais & $\begin{array}{l}\text { Atividades } \\
\text { passadas } \\
\text { presentes e } \\
\text { futuras }\end{array}$ & Divergente \\
\hline \multirow[t]{2}{*}{ Mobilidade } & Físico & $\begin{array}{l}\text { Habilidade } \\
\text { sensoriais }\end{array}$ & Convergente \\
\hline & Relações sociais & Morte e morrer & Divergente \\
\hline \multirow[t]{2}{*}{ Autocuidado } & Físico & Autonomia & Convergente \\
\hline & Meio ambiente & Intimidade & Divergente \\
\hline \multirow[t]{2}{*}{$\begin{array}{l}\text { Relações } \\
\text { Interpessoais }\end{array}$} & Relações sociais & $\begin{array}{l}\text { Participação } \\
\text { social }\end{array}$ & Convergente \\
\hline & Psicológico & $\begin{array}{l}\text { Habilidades } \\
\text { sensoriais }\end{array}$ & Divergente \\
\hline \multirow{2}{*}{$\begin{array}{l}\text { Atividades } \\
\text { de vida }\end{array}$} & Físico & Autonomia & Convergente \\
\hline & Relações sociais & Intimidade & Divergente \\
\hline \multirow[t]{2}{*}{ Participação } & Meio ambiente & $\begin{array}{l}\text { Participação } \\
\text { social }\end{array}$ & Convergente \\
\hline & Intimidade & Morte e morrer & Divergente \\
\hline
\end{tabular}

Para essa propriedade de medida, utilizamos o coeficiente de correlação de Spearman, em que: r=0,10 a 0,39: correlação fraca; r=0,40 a 0,69: correlação moderada; $\mathrm{r} \geq 0,70$ : correlação forte ${ }^{19}$.

\section{Análise estatística}

Para descrever as características clínicas, sociodemográficas e funcionais dos participantes do estudo, foram utilizadas as seguintes medidas descritivas: medidas de tendência central (média) e dispersão (desviopadrão). A distribuição dos dados foi verificada pelo teste de Shapiro-Wilk. A consistência interna foi atribuída de acordo com o alfa de Cronbach resultante da análise dos domínios do WHODAS 2.0. A confiabilidade teste-reteste foi analisada através do teste de correlação de Spearman entre os valores dos domínios e total do WHODAS 2.0 encontrados no teste-reteste. Para avaliar a validade de critério, foi utilizado o teste correlação de Spearman entre os domínios e pontuação total do WHODAS 2.0 e os questionários WHOQoL-bref e WHOQoL-old.

As análises estatísticas foram realizadas utilizando o programa Stata 13. Utilizou-se o nível de significância estatística de $\mathrm{p}<0,05$. 


\section{RESULTADOS}

\section{Caracterização da amostra}

Os idosos elegíveis para o estudo eram 290. Desses, 103 idosos recusaram-se a participar; 34 apresentaram comprometimento funcional grave; 9 tinham dificuldade de comunicação; 7. dificuldade de audição; 31 idosos não atingiram o ponto de corte esperado, o que evidenciava comprometimento cognitivo; e 6 idosos foram excluídos pois não realizaram a reaplicação (por desistência, piora do estado geral ou óbito) após sete dias. Contudo, foi possível obter a amostra de 100 participantes.

Os participantes eram predominantemente do sexo masculino (54) com idade média acima dos 70 anos $(74,7)$. Em relação à escolaridade: 20 eram analfabetos (MEEM=20,05), 37 tinham escolaridade de um a três anos (MEEM=23,62), 31 com escolaridade de quatro a sete anos (MEEM=24,7), 10 tinham escolaridade de oito a 10 anos $(\mathrm{MEEM}=26,4)$ e dois idosos com escolaridade de mais de 11 anos (MEEM=29). Dentre os domínios do WHODAS, aquele em que os idosos apresentaram maior pontuação foi o de "mobilidade". Os domínios em que obtiveram os menores escores e, consequentemente, apresentaram menor qualidade, foram os de "meio ambiente" do WHOQoL-bref, bem como o de “intimidade" do WHOQoL-old. As demais características estão registradas na Tabela 2.

Tabela 2. Descrição da amostra do estudo

\begin{tabular}{|c|c|c|}
\hline Variável & \multicolumn{2}{|c|}{ n (100) } \\
\hline \multicolumn{3}{|l|}{ Gênero } \\
\hline Masculino & \multicolumn{2}{|c|}{54} \\
\hline Feminino & \multicolumn{2}{|c|}{46} \\
\hline \multicolumn{3}{|l|}{ Estado civil } \\
\hline Nunca se casou & \multicolumn{2}{|c|}{32} \\
\hline Atualmente casado(a) & \multicolumn{2}{|c|}{6} \\
\hline Separado(a) & \multicolumn{2}{|c|}{7} \\
\hline Divorciado(a) & \multicolumn{2}{|c|}{23} \\
\hline Viúvo(a) & \multicolumn{2}{|c|}{32} \\
\hline \multicolumn{3}{|l|}{ Atividade de trabalho } \\
\hline Aposentado & \multicolumn{2}{|c|}{98} \\
\hline \multirow[t]{2}{*}{ Outros } & \multicolumn{2}{|c|}{2} \\
\hline & Média & Desvio-Padrão \\
\hline Faixa etária & 74,77 & 9,77 \\
\hline Anos de estudo & 3,72 & 2,96 \\
\hline
\end{tabular}

(continua)
Tabela 2. Continuação

Variável

n (100)

WHODAS 2.0 (versão 36 itens)

$\begin{array}{lrr}\text { Cognição } & 24,2 & 22,45 \\ \text { Mobilidade } & 42,06 & 32,56 \\ \text { Autocuidado } & 22 & 25,89 \\ \text { Relações interpessoais } & 24,83 & 24,38 \\ \text { Atividades de vida (domésticas) } & 16,20 & 15,40 \\ \text { Participação } & 30,41 & 21,87 \\ \text { TOTAL } & 30,36 & 19,71\end{array}$

WHOQOL-bref

$\begin{array}{lll}\text { Físico } & 59,16 & 18,77\end{array}$

$\begin{array}{lll}\text { Psicológico } & 60,20 & 18,87\end{array}$

Relações sociais $\quad 55,33 \quad 19,45$

Meio ambiente $\quad 54,30 \quad 17,58$

$\begin{array}{lll}\text { TOTAL } & 57,25 & 14,09\end{array}$

WHOQOL-old

Habilidades sensoriais $\quad 66 \quad 25,25$

$\begin{array}{lll}\text { Autonomia } & 47,53 & 21,11\end{array}$

Atividades passadas, presentes $\quad 58,68 \quad 20,56$

e futuras

$\begin{array}{lrr}\text { Participação Social } & 57 & 18,59 \\ \text { Morte e Morrer } & 73,37 & 24,30\end{array}$

Intimidade $\quad 40,24 \quad 28,14$

$\begin{array}{lll}\text { TOTAL } & 57,13 & 15,19\end{array}$

\section{Propriedades de medida}

A Tabela 3 apresenta os resultados da consistência interna e confiabilidade teste-reteste. Para a avaliação de consistência interna, o alfa de Cronbach variou de 0,75 a 0,94 entre as análises de seus domínios, demonstrando consistência interna satisfatória. $\mathrm{O}$ menor valor de consistencia interna foi encontrado no domínio "relações interpessoais"; posteriormente, realizou-se a análise dessa propriedade de medida ao ser excluída a questão referente à atividade sexual, obtendose um maior coeficiente alfa de Cronbach $(0,79)$. Além disso, os coeficientes de correlação de Spearman foram calculados para examinar a confiabilidade teste-reteste. Sua análise verificou forte estabilidade em todas as áreas do WHODAS $2.0(\geq 0,81)$, destacando-se a mobilidade $(\mathrm{r}=0,90)$, o autocuidado $(\mathrm{r}=0,91)$ e as atividades de vida $(r=0,89)$ como os valores mais elevados. Esses resultados indicam boa confiabilidade ao utilizar o instrumento para a população de idosos institucionalizados. 
Tabela 3. Distribuição dos coeficientes alfa de Cronbach e de correlação de Spearman de acordo com os domínios WHODAS 2.0

\begin{tabular}{|c|c|c|}
\hline WHODAS 2.0 (domínios) & $\begin{array}{l}\text { Consistência } \\
\text { interna (alfa de } \\
\text { Cronbach) }\end{array}$ & $\begin{array}{l}\text { Confiabilidade } \\
\text { teste-reteste }(r)\end{array}$ \\
\hline Cognição & 0,83 & 0,86 \\
\hline Mobilidade & 0,89 & 0,90 \\
\hline Autocuidado & 0,76 & 0,91 \\
\hline Relações interpessoais & 0,75 & 0,81 \\
\hline $\begin{array}{l}\text { Atividades de vida } \\
\text { (domésticas) }\end{array}$ & 0,94 & 0,89 \\
\hline Participação & 0,81 & 0,85 \\
\hline TOTAL & 0,93 & 0,95 \\
\hline
\end{tabular}

Cl: consistência interna; r: coeficiente de correlação de Spearman.

*Os domínios para atividades escolares ou do trabalho não foram analisados.

$\mathrm{Na}$ Tabela 4 estão os coeficientes de correlação para os domínios do WHODAS 2.0 e os domínios dos demais instrumentos, juntamente com seus respectivos níveis de significância estatística. Quanto às validades de critério convergentes e divergentes, as correlações foram de $r=0,40$ a 0,$68 ; p<0,0001$ e $r=0,19$ a 0,38 ; $\mathrm{p}=0,0001$ a $\mathrm{p}=0,004$, respectivamente.

\section{DISCUSSÃO}

OWHODAS é um instrumento traduzido e adaptado que demonstra consistência ao avaliar funcionalidade e incapacidade seguindo o modelo proposto pela $\mathrm{CIF}^{8}$. Apesar da abundância de ferramentas que avaliam a capacidades funcionais em diversas condições de saúde, havia poucos instrumentos para a avaliação da funcionalidade de idosos institucionalizados que incluíssem os domínios da condição de saúde, da função e estrutura do corpo e dos fatores pessoais ${ }^{10}$, seguindo o proposto pela CIF. A finalidade do presente estudo foi analisar algumas propriedades de medida da versão brasileira de 36 itens do WHODAS 2.0 em idosos institucionalizados.

Tabela 4. Matriz do coeficiente de correlação entre os domínios WHODAS 2.0, WHOQoL-bref e WHOQoL-old

\begin{tabular}{|c|c|c|c|c|c|c|c|}
\hline \multirow[b]{2}{*}{ Instrumentos/domínios } & \multicolumn{7}{|c|}{ WHODAS 2.0 / domínios } \\
\hline & Cognição & Mobilidade & Autocuidado & $\begin{array}{c}\text { Relações } \\
\text { Interpessoais }\end{array}$ & $\begin{array}{l}\text { Atividades de } \\
\text { vida }\end{array}$ & Participação & Total \\
\hline \multicolumn{8}{|l|}{ WHOQOL-bref } \\
\hline Físico & $-0,45^{*}$ & $-0,52^{*}$ & $-0,57^{*}$ & $-0,48^{*}$ & $-0,46^{*}$ & $-0,64^{*}$ & $-0,69 *$ \\
\hline Psicológico & $-0,49^{*}$ & $-0,19$ & $-0,33^{*}$ & $-0,32^{*}$ & $-0,31^{*}$ & $-0,51^{*}$ & $-0,46^{*}$ \\
\hline Relações sociais & $-0,25^{*}$ & $-0,22^{*}$ & $-0,14$ & $-0,34^{*}$ & $-0,38^{*}$ & $-0,37^{*}$ & $-0,39 *$ \\
\hline Meio ambiente & $-0,28^{*}$ & $-0,11$ & $-0,17$ & $-0,27^{*}$ & $-0,10$ & $-0,49^{*}$ & $-0,34^{*}$ \\
\hline Total & $-0,48^{*}$ & $-0,33^{*}$ & $-0,40^{*}$ & $-0,46^{*}$ & $-0,40^{*}$ & $-0,66^{*}$ & $-0,62^{*}$ \\
\hline \multicolumn{8}{|l|}{ WHOQoL-old } \\
\hline Habilidades sensoriais & $-0,46^{*}$ & $-0,41^{*}$ & $-0,30^{*}$ & $-0,38^{*}$ & $-0,27^{*}$ & $-0,43^{*}$ & $-0,50 *$ \\
\hline Autonomia & $-0,40^{*}$ & $-0,20^{*}$ & $-0,16$ & $-0,33^{*}$ & $-0,21^{*}$ & $-0,45^{*}$ & $-0,40 *$ \\
\hline $\begin{array}{l}\text { Atividades passadas, } \\
\text { presentes e futuras }\end{array}$ & $-0,24^{*}$ & $-0,20^{*}$ & $-0,12$ & $-0,37^{*}$ & $-0,27^{*}$ & $-0,43^{*}$ & $-0,35^{*}$ \\
\hline Participação Social & $-0,27^{*}$ & $-0,51^{*}$ & $-0,25^{*}$ & $-0,44^{*}$ & $-0,31^{*}$ & $-0,58^{*}$ & $-0,57^{*}$ \\
\hline Morte e Morrer & $-0,19^{*}$ & $-0,11$ & $-0,22^{*}$ & $-0,24^{*}$ & $-0,26^{*}$ & $-0,37^{*}$ & $-0,30 *$ \\
\hline Intimidade & $-0,21^{*}$ & $-0,26^{*}$ & $-0,12$ & $-0,22^{*}$ & $-0,36^{*}$ & $-0,23^{*}$ & $-0,33^{*}$ \\
\hline Total & $-0,44^{*}$ & $-0,42^{*}$ & $-0,28^{\circ}$ & $-0,48^{*}$ & $-0,42^{*}$ & $-0,61^{*}$ & $-0,61^{*}$ \\
\hline
\end{tabular}

Os resultados do presente estudo mostraram propriedades de medida satisfatórias, que permitem o uso da ferramenta para avaliar e acompanhar a funcionalidade de idosos institucionalizados. Mesmo não sendo objetivo deste estudo, ao se comparar os domínios, a "mobilidade" apresentou escore mais elevado, indicativo de maior dificuldade apresentada por essa população, o que converge com outros estudos envolvendo idosos institucionalizados e que revelam o prejuízo na locomoção e o alto risco de quedas como as alterações mais presentes nesses idosos ${ }^{3}$. Reitera-se, assim, a importância do WHODAS 2.0 na avaliação da funcionalidade de idosos institucionalizados.

Em relação às propriedades de medidas, principal objetivo deste estudo, valores que determinam boa consistência interna foram encontrados em todos os domínios e no valor total do WHODAS 2.0. Em dois 
estudos brasileiros de validação em indivíduos com doença pulmonar obstrutiva crônica ${ }^{11} \mathrm{e}$ em pessoas com chikungunya ${ }^{20}$, foram obtidos valores similares, com alfa de Cronbach de 0,72 a 0,89 nos domínios e de 0,85 a 0,97 no valor total.

Forte confiabilidade teste-reteste também foi encontrada em todos os domínios, aferindo estabilidade quando aplicados em momentos diferentes. Os valores corroboram com os achados do estudo de Santos et al. ${ }^{21}$, estudo brasileiro de validação do WHODAS para a população com fibromialgia, que obteve valores de $r=0,64$ a $0,94^{21}$, bem com os valores encontrados na pesquisa de Silveira et al. ${ }^{22}$, estudo brasileiro de validação para a população com cegueira, que apresentou intervalo de valores de $r$ entre 0,64 a 0,94 para a confiabilidade teste-reteste ${ }^{22}$.

Ao analisar o domínio "relações interpessoais", os resultados para consistência interna e confiabilidade teste-reteste deste domínio foram inferiores quando comparados às mesmas propriedades de outros domínios, ainda que dentro dos valores adequados que garantem confiabilidade. Durante as entrevistas, a maioria dos idosos manifestou insatisfação com relação à socialização e às relações interpessoais, o que corrobora com o encontrado na literatura em estudo também realizado com idosos institucionalizados ${ }^{1}$. Alguns participantes relataram dificuldade para responder à questão referente às atividades sexuais. Ao se avaliar a consistência interna sem a referida questão, os resultados indicaram valor mais elevado de alfa de Cronbach (0,79). Esse achado corrobora com o estudo de Ćwirlej-Sozańska et al. ${ }^{23}$ que, ao avaliar a validade teórica das questões estimando os itens que compõem os próprios domínios para a população de idosos não residentes de ILPI, encontrou resultado inferior no item referente à atividade sexual, o que pode justificar a consistência interna inferior encontrada no presente estudo e que pode ter favorecido maior variação na pontuação teste-reteste.

A confiabilidade teste-reteste do domínio do "autocuidado" manteve-se como uma das mais altas, o que garante estabilidade; porém, sua consistência interna, mesmo que adequada, mostrou-se inferior quando comparada a outros domínios. Tendo como exemplo o item que aborda a "alimentação", em todas as ILPI em que os questionários foram aplicados, o alimento era preparado e servido mesmo àqueles idosos que apresentavam condições físicas e cognitivas de se servirem. Entretanto, idosos dependentes ou não, escolhiam menor grau de dificuldade em suas respostas, já que eram realizadas sem dificuldades. Isso pode justificar o escore relativamente baixo de dificuldade (média $=22 ; \mathrm{DP}=25,89$ ), acompanhado por uma consistência interna inferior e com alta confiabilidade teste-reteste. Em um estudo brasileiro de validação para a população com HIV/AIDS, foi obtida consistência interna abaixo dos parâmetros adequados nesse mesmo domínio $(0,32)$, no qual a confiabilidade teste-reteste não foi analisada ${ }^{24}$.

$\mathrm{Na}$ análise de validade convergente e divergente foram encontrados apenas valores de coeficientes de correlação negativos. Isso ocorreu pois os instrumentos utilizados para análise dos dados possuem pontuação inversa. No que se refere à intensidade da relação dos coeficientes, identificou-se apenas correlações moderadas entre os instrumentos, mesmo resultado que fora encontrado em outros estudos que também utilizaram o WHOQol-bref ${ }^{22,25}$.Em um estudo realizado na Polônia com a população idosa (60-70 anos) e que utilizou o mesmo instrumento para apoiar a validade de critério, a correlação mais alta ao correlacionar os domínios foi de $0,73^{23}$. Em nosso estudo encontramos um valor inferior, mas próximo de valores de outros estudos com o mesmo objetivo $^{20,25}$. No estudo de validação do WHODAS para uso em pessoas pós-chikungunya ${ }^{20}$, foi obtida forte correlação do valor total do WHODAS com o domínio "físico" do WHOQoL-bref; em nosso estudo, obtivemos correlação moderada $(r=-0,69)$, o mesmo valor encontrado em outros estudos com a população brasileira $^{22,25}$. Nenhum outro estudo publicado utilizou WHOQol-old para a análise da validade de critério do instrumento WHODAS.

Esperava-se que os domínios "mobilidade, "autocuidado" ou "atividades de vida" do WHODAS obtivessem, no mínimo, correlação moderada com o domínio "autonomia" do WHOQoL-old. Entretanto, as quatro questões relativas à "autonomia" tem relação com a liberdade de tomar decisões e de fazer o que gosta e com a habilidade de poder controlar o futuro ${ }^{16}$, enquanto o WHODAS, em seu domínio "mobilidade", avalia a dificuldade em atividades como ficar em pé, movimentar-se, sair de casa e caminhar longas distâncias. Já o "autocuidado" avalia itens como lidar com a própria higiene, vestir-se, comer e permanecer sozinho; e, por fim, "atividades de vida" aborda as responsabilidades domésticas ${ }^{9}$. Essas constatações podem ser justificativas para as correlações fracas entre "autonomia" "mobilidade", "autocuidado" e "atividades de vida", já que ambos instrumentos abordam de formas diferentes os domínios citados. Cabe ressaltar que o domínio "autonomia" do 
WHOQoL-old obteve correlação moderada com o domínio "cognição" do WHODAS. A "cognição" avalia compreensão e comunicação, variáveis que interferem na capacidade de decisão do indivíduo e, consequentemente, na sua capacidade de comandar as ações, ou seja, na manutenção de sua autonomia ${ }^{2}$, outro achado que justifica a validade do instrumento aqui avaliado.

Quando analisado o domínio de "relações interpessoais" do WHODAS, obtivemos correlação moderada com o domínio "participação social" do WHOQol-old e correlação fraca com o domínio "relações sociais" do WHOQol-bref. Em estudos brasileiros de validação do WHODAS que adotaram o WHOQol-bref para a validade de critério, foi obtida correlação moderada ao correlacionar esses domínios para a população com chikungunya ${ }^{20}$. No entanto, tanto no trabalho de validação para pacientes em hemodiálise ${ }^{25}$ quanto para pessoas com cegueira ${ }^{22}$, foram descritas apenas correlações fracas. Apesar de bem similares em suas avaliações, o WHOQoL-bref analisa como o indivíduo se sente no tempo presente, a respeito de suas relações pessoais, seu suporte social e sua atividade sexual ${ }^{16}$; já o WHODAS avalia a dificuldade do indivíduo de interagir com outras pessoas nos últimos 30 dias $^{9}$, o que pode ser uma justificativa para a fraca correlação.

Outras correlações foram encontradas para apoiar a validade convergente. Castro et al..$^{25}$ validou a versão de 36 itens do WHODAS em pacientes em hemodiálise utilizando o WHOQoL-bref em sua análise e, assim como em nosso estudo, encontrou correlações moderadas do valor total do WHODAS com os valores totais dos outros dois instrumentos utilizados para assegurar a validade convergente. Vale ressaltar que alguns estudos utilizam o instrumento de avaliação de qualidade de vida Medical Outcomes Study 36-Item Short-Form Health Survey (SF-36) para analisar sua validade de critério, como no estudo de Garin et al. ${ }^{12}$. Nessa pesquisa, foram avaliadas as propriedades de medida do WHODAS 2.0 para pacientes de sete centros da Europa com diferentes condições crônicas. Os autores acharam correlações moderadas $(\mathrm{r}=-0,63)$, com exceção apenas do domínio "mobilidade", que obteve correlação forte $(\mathrm{r}=-0,75)$ com o domínio "capacidade funcional" do SF-36. Isso também foi encontrado no estudo de pacientes com fibromialgia que utilizou o SF-36 na análise e obteve correlação forte com o domínio "mobilidade" $(\mathrm{r}=-0,72)$, seguido por correlações moderadas com os demais domínios $(\mathrm{r}=-0,69)^{21}$. De forma geral, resultados semelhantes foram encontrados para a validade convergente utilizando esses dois instrumentos.
Para apoiar a validade divergente, obtivemos resultados que apontam que os domínios que deveriam divergir entre si obtiveram correlações fracas. Resultados similares foram relatados no estudo de validação para pessoas em hemodiálise, que avaliou a validade divergente de forma semelhante ${ }^{25}$.

Como limitação do estudo podemos destacar o fato de a coleta de dados ter sido realizada em dois períodos distintos, entre 2016 e 2017 e em 2019, e a incapacidade de realizar a pesquisa em apenas um local, pois não havia o número de idosos elegíveis em uma única instituição. Entretanto, a coleta atingiu 100 participantes, o que nos permitiu a obtenção de um intervalo de confiança de $95 \%$ para a validação do instrumento ${ }^{14}$. Ainda, apesar de o instrumento ser estável e confiável em sua avaliação generalizada, evidenciamos a necessidade de planejamento e cuidado no uso da questão referente a atividades sexuais na aplicação para essa população. Apenas correlações moderadas foram encontradas para apoiar a validade de critério convergente. Tais correlações eram esperadas, já que ambos se comunicam e são convergentes entre si, porém avaliam de forma diferente constructos similares ${ }^{9}$. Além disso, estudos que utilizaram o instrumento SF-36 também obtiveram, em sua grande maioria, correlações moderadas. Entretanto, outras estratégias poderiam ter sido utilizadas, como a aplicação concomitantemente do questionário de bem estar subjetivo (WHOQoL) com outras ferramentas que avaliem a funcionalidade, como a escala de Lawton e Brody e a medida de independência funcional (MIF), que poderiam ser correlacionados com os domínios do autocuidado e das atividades de vida, ou o teste timed up and go (TUG), que poderia ser correlacionado com o domínio da mobilidade.

Deve-se destacar que este estudo disponibiliza uma ferramenta genérica com suas propriedades de medida testadas e consistentes baseada na CIF que visa avaliar a funcionalidade de pessoas com diversas condições de saúde e deficiências. A disponibilização dessa ferramenta permitirá seu uso na área acadêmica e poderá ser incorporado às práticas clínicas dos profissionais de saúde, auxiliando no acompanhamento do idoso ao longo do período de institucionalização e proporcionando uma abordagem mais abrangente e direcionada de cuidados. Além disso, este estudo possibilita utilizar a funcionalidade como indicador de saúde para essa população. Esse constructo não podia ser aferido até agora, pois não existiam ferramentas que realizassem essa abordagem seguindo o modelo biopsicossocial. 


\section{CONCLUSÃO}

Este estudo demonstrou propriedades de medida (consistência interna, confiabilidade teste reteste, validade de critério convergente e divergente) do WHODAS apropriadas, tornando-o válido e confiável para avaliar a funcionalidade em idosos institucionalizados.

\section{AGRADECIMENTOS}

Os autores agradecem aos idosos, aos cuidadores e aos profissionais pela colaboração com o estudo e aos colegas da residência multiprofissional e da graduação da UFTM por todo o suporte técnico e científico.

\section{REFERÊNCIAS}

1. Haddad PCMB, Calamita Z. Sociodemographic aspects, quality of life and health of the institutionalized elderly. Rev Enferm UFPE on line. 2020;14:e2343416. doi: 10.5205/1981-8963.2020.243416

2. Oliveira MR, Konzen VM, Fleig TCM, Signori LU. Impact on functional and cognitive capacity in elderly after a year of institutionalization. Fisioter Bras 2019;20(2):139-46. doi: 10.33233/fb.v20i2.2792

3. Reis CCA, Menezes TMO, Freitas AVF, Pedreira LC, Freitas RA, Pires IB. Ser-pessoa-idosa institucionalizada : sentido do vivido à luz da fenomenologia Heideggeriana. Rev Bras Enferm. 2019;72(6):1710-6. doi: 10.1590/0034-7167-2018-0763

4. Lopes VM, Scofield AMTS, Alcântara RKL, Fernandes BKC, Leite SFP, Borges CL. What taken the elderly people to institutionalization? Rev Enferm UFPE on line. 2018;12(9):242835. doi: 10.5205/1981-8963-v12i9a234624p2428-2435-2018

5. Fluetti MT, Fhon JRS, Oliveira AP de, Chiquito LMO, Marques $S$. The frailty syndrome in institutionalized elderly persons. Rev Bras Geriatr Gerontol. 2018;21(1):60-9. doi: 10.1590/1981-22562018021.170098

6. Damo CC, Doring M, Alves ALS, Portella MR. Risk of malnutrition and associated factors in institutionalized elderly persons. Rev Bras Geriatr Gerontol. 2018;21(6):711-7. doi:10.1590/1981-22562018021.180152.8

7. Castaneda L, Bergmann A, Bahia L. A Classificação Internacional de Funcionalidade, Incapacidade e Saúde: Uma revisão sistemática de estudos observacionais. Rev Bras Epidemiol. 2014;17(2):437-51. doi: 10.1590/1809-4503201400020012ENG

8. Castro SS, Leite CF. Translation and cross-cultural adaptation of the World Health Organization Disability Assessment Schedule - WHODAS 2.0. Fisioter Pesqui. 2017;24(4):385-91. doi: 10.1590/1809-2950/17118724042017

9. Castro S, Leite C. Manual do WHO Disability Assessment Schedule WHODAS 2.0 [Internet]. Uberaba: Universidade Federal do Triângulo Mineiro; 2015 [cited 2020 May 10]. Available from: https://apps. who.int/iris/bitstream/handle/10665/43974/9788562599514 por.pdf; j s e s s i on i d = E O 8 A 919 CD 8 E $520 \overline{2}$ 3CA1E0028B34B31A1? sequence $=19$

10. Gomes CS, Buranello MC, Castro SS. Assessment instruments of functioning in Brazilian elderly and the ICF: a systematic review. Fisioter Mov. 2017;30(3):625-37. doi: 10.1590/19805918.030.003.AR03

11. Zacarias LC, Câmara KJ da C, Alves BM, Castro SS, Leite CF. Validation of the Brazilian version of World Health Organization Disability Assessment Schedule (WHODAS 2.0) in individuals with COPD [Internet]. Fortaleza: Universidade Federal do Ceará; 2019 [cited 2020 May 10]. Available from: http://www.repositorio.ufc.br/bitstream/riufc/48730/1/2019_ art_lczacarias.pdf

12. Garin O, Almansa J, Nieto M, Chatterji S, Vilagut G, Alonso J, et al. Validation of the "World Health Organization Disability Assessment Schedule, WHODAS-2" in patients with chronic diseases. Health Qual Life Outcomes. 2010;19(8):51. doi: 10.1186/1477-7525-8-51

13. Souza AC, Alexandre NMC, Guirardello EB. Propriedades psicométricas na avaliação de instrumentos: avaliação da confiabilidade e da validade. Epidemiol Serv Saude. 2017;26(3):649-59. doi: 10.5123/s1679-49742017000300022

14. Bland JM, Altman DG. Statistical methods for assessing agreement between two methods of clinical measurement. Lancet. 1986 Feb;1(8476):307-10.

15. Caramelli P, Nitrini R. Como avaliar de forma breve e objetiva o estado mental de um paciente? Rev Ass Med Bras [Internet]. 2000 [cited 2020 May 10];46(4):289-311. Available from: https:// www.scielo.br/pdf/ramb/v46n4/3765.pdf

16. Fleck MPA, Louzada S, Xavier M, Chachamovich E, Vieira G, Santos L, et al. Aplicação da versão em português do instrumento abreviado de avaliação da qualidade de vida “WHOQOL-bref." Rev Saude Publica. 2000;34(2):178-83. doi: 10.1590/S0034-89102000000200012

17. Fleck MPA, Chachamovich E, Trentini CM. Desenvolvimento e validação da versão em Português do módulo WHOQOLOLD. Rev Saude Publica. 2006;40(5):7. doi: 10.1590/ S0034-89102006000600007.

18. Bot SDM, Terwee CB, Van Der Windt DAWM, Bouter LM, Dekker J, De Vet HCW. Clinimetric evaluation of shoulder disability questionnaires: A systematic review of the literature. Ann Rheum Dis. 2004;63(4):335-41. doi: 10.1136/ard.2003.007724.

19. De Campos MCT, Marziale MHP, Santos JLF. Cross-cultural adaptation and validation of the world health organization health and work performance questionnaire to brazilian nurses. Rev Esc Enferm. 2013;47(6):1338-44. doi: 10.1590/ S0080-623420130000600013

20. Sousa AJS, Silva MC, Barreto MCA, Nunes BP, Coutinho BD, Castro SS. Propriedades psicométricas do WHODAS para uso em pessoas com chikungunya no Brasil. Fisioter Pesqui. 2019;26(4):419-26. doi: 10.5123/s1679-49742017000300022

21. Santos AEN, Barreto MCA, Castro SS, Leite CF, Moraleida FRJ, Nunes ACL. Validação do Questionário Whodas 2.0 (Brasil) para indivíduos com fibromialgia: dados parciais. Proceedings of the 3rd Congresso Brasileiro e Internacional da Associação Brasileira de Fisioterapia Traumato-Ortopédica [Internet]; 2019 May 23-25; Belo Horizonte, Brazil. Uberaba: Universidade Federal do Triângulo 
Mineiro; 2019 [cited 2020 May 10]. Available from: http://seer.uftm. edu.br/anaisuftm/index.php/abrafito/article/view/2359.

22. Silveira LS, Castro SS, Leite CF, Oliveira NML, Salomão AE, Pereira K. Validade e confiabilidade da versão brasileira do World Health Organization Disability Assessment Schedule em pessoas com cegueira. Fisioter Pesqui. 2019;26(1):22-30. doi: 10.1590/1809-2950/17013126012019

23. Ćwirlej-Sozańska A, Wilmowska-Pietruszyńska A, Sozański B. Validation of the Polish version of the World Health
Organization Disability Assessment Schedule (WHODAS 2.0) in an elderly population (60-70 years old). Int J Occup Saf Ergon. 2018;24(3):386-94. doi: 10.1080/10803548.2017.1316596

24. Barbosa KSS, Castro SS, Leite CF, Nacci FR, Accioly MF. Validation of the Brazilian version of the world health organization disability assessment schedule 2.0 for individuals with HIV/AIDS. Cienc Saude Colet. 2020;25(3):837-44. doi: 10.1590/1413-81232020253.18992018

25. Castro SS, Leite CF, Baldin JE, Accioly MF. Validation of the Brazilian version of WHODAS 2.0 in patients on hemodialysis therapy. Fisioter Mov. 2018;31:e003130. doi: 10.1590/1980-5918.031.AO30 